\title{
Surface-Sandwich Segregation Phenomena in Bimetallic Ag-Ni and Pd-Ni Nanoparticles: A Molecular Dynamics Study
}

\author{
E.V. Levchenko ${ }^{1, a}$, A.V. Evteev ${ }^{1, b}$, I.V. Belova ${ }^{1, c}$ and G.E. Murch ${ }^{1, d}$ \\ ${ }^{1}$ The University Centre for Mass and Thermal Transport in Engineering Materials, \\ School of Engineering, The University of Newcastle, Callaghan, NSW 2308, Australia \\ aElena.Levchenko@newcastle.edu.au, bAlexander.Evteev@newcastle.edu.au, \\ 'Irina.Belova@newcastle.edu.au, dGraeme.Murch@newcastle.edu.au
}

Keywords: Molecular dynamics, embedded atom method, interdiffusion, surface segregation, silver, palladium, nickel, alloy nanoparticles.

\begin{abstract}
By molecular dynamics simulation it is shown that interdiffusion in the initial f.c.c. Agcore $(\approx 28$ at. $\%)-\mathrm{Ni}$-shell $(\approx 72$ at. $\%)$ and Ni-core $(\approx 34$ at. $\%)-\mathrm{Pd}$-shell $(\approx 66$ at. $\%)$ nanoparticles can lead to surface-sandwich segregation. It is observed that there is a separation of the initial Ag-Ni core-shell structure into Ag-core - Ni-intermediate shell - Ag-disperse surface monolayer. The initial crystal Ni-Pd core-shell structure transforms to the core of a non-crystalline Pd-rich solid solution with quite strongly developed icosahedral short-range order, which is covered by a surface-sandwich shell, where $\mathrm{Ni}$ atoms are located in the centres of interpenetrating icosahedra of a subsurface Kagomé net layer while the Pd atoms occupy the vertices of the icosahedra and cover this Ni layer from inside and outside. We demonstrate that under certain conditions a surface-sandwich segregation phenomenon at the nanoscale can be observed in systems with completely different phase diagrams in the bulk states: in systems displaying the extremely low mutual solubility as in the Ag-Ni system, or in systems exhibiting a continuous mutual solid solubility like the Pd-Ni system.
\end{abstract}

\section{Introduction}

Nanoparticles are very promising materials for advanced applications, because they often exhibit specific structures and properties at the nanoscale, which cannot be observed in bulk materials. In particular, the nanoparticles of noble metals have attracted much attention due to their wide applicability in catalysis and nanotechnology. More recently, particular interest has focused on bimetallic nanoparticles, which compared to monometallic nanomaterials, show even more remarkable characteristics in catalytic, optical, electrical, magnetic properties and in some combinations of the above properties see [1] and references therein. Bimetallic nanoparticles exhibit the characteristics that are not just the addition of two properties of the constituent monometallic nanoparticles see [2] and references therein. Indeed, the properties of bimetallic nanoparticles depend not only on the size, as in pure nanoparticles, but also strongly on composition, as for example this was demonstrated for catalytic activity [1]. In general, at the nanoscale, bimetallic clusters can form various kinds of structure: from a high entropy situation of a solid solution random alloy to such a low entropy product as cluster in a cluster, a layered compound or a core shell structure see [1] and references therein. As an empirical law, to minimise the total energy, one expects the component with the lower surface energy to accumulate preferentially at the surface. However such factors as the lattice mismatch between two components, phase diagrams, production method, and surrounding media could modify such a simple empirical rule [3].

Today, noble metals are the most important catalysts for a range of existing and potential catalytic reactions, including hydrogenation and oxidation reactions, carbon-carbon coupling reactions, electrocatalytic reactions for low-temperature fuel cells and many others [4]. Noble-metal nanoparticles as a single active metal component in catalysis have received considerable attention on account of their remarkable activity and efficiency but at the same time they have negative economic aspects because of their high cost. Since catalytic reactions occur on the surface of the nanoparticles, a large fraction of metal in the core of the nanoparticle is in fact wasted. This issue becomes very important for nanoparticles of expensive noble metals such as palladium and silver. An interesting approach starts by alloying Ag and Pd with a lower cost and higher surface energy metal (for example, Ni), since it would 
then be economically attractive to design bimetallic catalyst nanoparticles in which the precious and catalytic Ag and Pd atoms segregate to the surface. Promising results in this sense have been obtained experimentally for bimetallic Ag-Ni [3,5-7] and Pd-Ni nanoparticles [8-14].

It is clear in the case of alloy nanoparticles, that surface segregation plays the major role for the understanding of their structure and functional properties. Furthermore, as was noted in [15], in materials with a large fraction of the atoms at surfaces the thermodynamic driving force towards surface segregation might be large. This may lead to excessive solid-state diffusion and hence possibly to an increased density of faults and defects in the crystal structure, moreover, bulk order may be broken at the grain boundaries and interfaces.

Silver and nickel are known to be immiscible metals and only recently has the Ag-Ni system been investigated widely at the nanoscale. The most experimental [3,5-7] and theoretical [2,16-19] studies of Ag-Ni nanoparticles by different methods and techniques have demonstrated that silver atoms generally lie on the surface while nickel atoms prefer to occupy the core of the nanoparticles. The previous theoretical studies mentioned above [2,16-19] have revealed some detailed structures of Ag-Ni nanoparticles, however only small sizes of nanoparticles (up to $\sim 400$ atoms) have been investigated.

The surface segregation phenomena at low indices surfaces in different Pd-Ni crystal systems have been studied by several experimental techniques [11-14,20-22] and by modelling with different theoretical approaches [11,15,23-29]. Most of the measurements and calculations showed a considerable segregation of $\mathrm{Pd}$ to the surfaces and oscillations in the atomic layer composition near the surfaces with the second layer enriched by $\mathrm{Ni}$ atoms.

In principle, the properties of alloy nanoparticles can be tuned by varying the composition, size, shape and atomic ordering, but on the other hand an efficient search of alloy compositions and technological ways of fabrication of a nanoparticle with a desirable structure and properties is a very complex problem requiring a deep physical understanding of the atomic processes and phenomena occurring at the nanoscale. Atomistic simulation techniques such as molecular dynamics (MD) have become a powerful tool in the field of nanotechnology by providing physical insight into understanding phenomena at an atomistic scale and predicting many of the properties of nanomaterials. In particular in the field of nano-catalysis the computational approach has been aptly named an "engine for catalyst design/search" [30].

In this work, we carry out a series of long-time scale MD simulations in combination with the embedded atom method (EAM) in order to investigate the process of surface segregation on the structure of bimetallic Ag-Ni and Pd-Ni nanoparticles of diameter of $\sim 4.5 \mathrm{~nm}$ (several thousand atoms) containing $\sim 72$ at. $\% \mathrm{Ni}$ and $\sim 34$ at. $\% \mathrm{Ni}$, respectively. The main reasons for choosing Ag$\mathrm{Ni}$ and $\mathrm{Pd}-\mathrm{Ni}$ nanoparticles are (i) they have been prepared and characterized by using experimental methods; (ii) they are very effective Pd- and Ag-based catalysts; and (iii) the ability to design a lower cost nano-catalyst. Some preliminary results of this study have been published recently in [31].

\section{The model}

The EAM represents the total energy of an atomic system in the form [32]:

$$
E_{\mathrm{tot}}=\sum_{i}\left[F_{i}\left(\bar{\rho}_{i}\right)+\frac{1}{2} \sum_{j(\neq i)} \phi_{i j}\left(r_{i j}\right)\right] \text {. }
$$

In this expression, $\bar{\rho}_{i}$ is the host electron density at atom $i$ due to the remaining atoms of the system, $F_{i}\left(\bar{\rho}_{i}\right)$ is the energy to embed atom $i$ into the background electron density $\bar{\rho}_{i}$ and $\phi_{i j}\left(r_{i j}\right)$ is a pair-interaction representing the core-core repulsion between atoms $i$ and $j$ separated by the distance $r_{i j}$. The electron density is approximated by the superposition of atomic densities:

$$
\bar{\rho}_{i}=\sum_{j(\neq i)} \rho_{j}\left(r_{i j}\right),
$$


where $\rho_{j}\left(r_{i j}\right)$ is the atomic electron density of atom $j$ at the distance $r_{i j}$ from the nucleus. The embedding function $F_{i}\left(\bar{\rho}_{i}\right)$ is universal in that it does not depend on the source of the background electron density. Thus the same embedding function is used to calculate the energy of an atom in an alloy that is used in the pure material. The form of the pair-repulsion term has been studied from first principles by Daw [33]. This analysis shows that the pair-interaction between two different species can be approximated by the geometric mean of the pair-interaction for the individual species. This observation, along with the Coulombic origin of the pair-interaction term, suggests writing the pair-interaction between atoms in terms of effective charges $Z_{i}\left(r_{i j}\right)$ as:

$$
\phi_{i j}\left(r_{i j}\right)=Z_{i}\left(r_{i j}\right) Z_{j}\left(r_{i j}\right) / r_{i j} \text {. }
$$

To apply this method, the embedding functions, pair-repulsions (or effective charges) and atomic electron densities must be known. For the Ag-Ni system we use the sets of EAM functions developed by Foiles, Baskes and Daw (EAM-FBD) [34] by fitting to bulk equilibrium solid properties (lattice constant, heat of sublimation, elastic constants, vacancy formation energy) of pure $\mathrm{Ag}$ and $\mathrm{Ni}$, and $\mathrm{Ag}-\mathrm{Cu}, \mathrm{Ag}-\mathrm{Au}, \mathrm{Ag}-\mathrm{Pd}, \mathrm{Ni}-\mathrm{Cu}, \mathrm{Ni}-\mathrm{Au}, \mathrm{Ni}-\mathrm{Pd}$ and $\mathrm{Ni}-\mathrm{Pt}$ binary alloy heats of solution. For Pd-Ni system we use the sets of EAM functions developed by Wilson and Bisson (EAM-WB) by fitting to bulk equilibrium solid properties of pure $\mathrm{Pd}$ and $\mathrm{Ni}$, and the hydrogen heat of solution in Pd and Ni.

The details of the parameterization of the sets of EAM-WB functions are presented in the Appendix of [35]. Furthermore, these sets of EAM-FBD and EAM-WB functions provided a good description of the liquid state of $\mathrm{Ni}, \mathrm{Ag}$ and $\mathrm{Pd}[35,36]$, and so must include reasonable values of the anharmonicity of the interatomic interactions.

Some very helpful qualitative predictions of the surface segregation behaviour in alloys are possible from a macroscopic thermodynamic point of view. In bimetallic systems, surface segregation phenomena must be driven by the differences in surface energy for various surface compositions. Therefore, the surface energies of the low indices faces (100), (110) and (111) of the pure metals can show that the use of the models is reasonable. The surface energies of the low indices faces of $\mathrm{Ag}$ and $\mathrm{Ni}$ with EAM-FBD potentials have been calculated in [34], while the surface energies of Pd and Ni with EAM-WB potentials have been presented in [31]. The surface energy is computed by minimizing the energy of a slab of atoms with respect to all atomic positions to incorporate surface relaxation effects. The surface energy is then the difference between the energy of the slab and that of an equal number of atoms in the bulk. The surface energies of $\mathrm{Ag}$ (EAM-FBD), Pd (EAM-WB) and Ni (EAM-FBD and EAM-WB) are collected in Table 1. In all cases, the close-packed (111) face has the lowest energy, followed by the (100) and (110) faces. This Table also contains the estimates of the average surface energy based on liquid metal surface energies from experimental data [37]. The experimental values have been crudely extrapolated from the melt temperature down to $0 \mathrm{~K}$. This agreement between theory and experiment is reasonable since there is the presence of less energetically favourable faces and defects on the average surface for which these estimates are intended. The theoretical values are for ideal low indices faces which one would expect to have a below-average surface energy. More importantly for segregation phenomena, the theory predicts the correct relative size of the surface energies for the metals [38].

Table 1. Surface energies of the low indices faces of Ag and Ni predicted by EAM-FBD potentials [34] as well as Pd and Ni predicted by EAM-WB potentials [31] in comparison with experimental data [37] in units of $\mathrm{J} / \mathrm{m}^{2}$.

\begin{tabular}{|c|c|c|c|c|c|c|c|}
\hline & \multicolumn{2}{|c|}{$\mathrm{Ag}$} & \multicolumn{2}{|c|}{$\mathrm{Pd}$} & \multicolumn{3}{|c|}{$\mathrm{Ni}$} \\
\hline & EAM-FBD & Exp. & EAM-WB & Exp. & EAM-FBD & EAM-WB & Exp. \\
\hline$(111)$ & 0.62 & \multirow{3}{*}{1.24} & 1.06 & \multirow{3}{*}{2.00} & 1.45 & 1.30 & \multirow{3}{*}{2.37} \\
\hline$(100)$ & 0.705 & & 1.25 & & 1.58 & 1.54 & \\
\hline$(110)$ & 0.77 & & 1.34 & & 1.73 & 1.70 & \\
\hline
\end{tabular}


The heats of solution and the deviations of the lattice constant from Vegard's law for bulk compositions of Ag-Ni and Pd-Ni systems have also been determined with EAM-FBD and EAMWB potentials, respectively, and compared with experimental data [39-41] where possible. The results of calculations for the heat of solution (i.e. the difference between the enthalpy of the alloy and the linear interpolation of the enthalpy of the pure metals) are given as the solid line in Fig. 1(a) for the Ag-Ni system and in Fig. 2(a) for the Pd-Ni system. For comparison, the experimental values for Ag-Ni [39] and Pd-Ni [40] are presented as open circles. Since the Ag-Ni system has extremely low mutual solubility, the experimental data for this system are very limited. In [39] the heat of mixing was reported of two supersaturated Ag-Ni solid solutions containing 5.8 and 9.6 at. $\% \mathrm{Ni}$, produced in thin-film form by electron-beam co-evaporation. The experimental results are in good agreement with calculated data at the same compositions; the deviation from each other does not exceed $0.012 \mathrm{eV} / \mathrm{at}$ (Fig. 1(a)). In general, the calculated heats of solution for Ag-Ni system are large positive (Fig. 1(a)) that is consistent with very little mutual solubility of $\mathrm{Ag}$ and $\mathrm{Ni}$ found in experiment [42,43]. For the Pd-Ni system the agreement between calculated and experimental heats of solution is also quite good; the deviation from each other at any composition does not exceed $0.025 \mathrm{eV} / \mathrm{at}$ (Fig. 2(a)). Furthermore, in all cases, the heat of solution is rather small. The small heat of solution is quite plausible, since $\mathrm{Pd}$ and $\mathrm{Ni}$ are neighbours in the periodic system and exhibit a continuous mutual solid solubility without atomic ordering. Fig. 1(b) shows the deviations of the lattice constant from Vegard's law calculated for Ag-Ni system. Unfortunately we could not find an experimental data on the lattice constants of $\mathrm{Ag}-\mathrm{Ni}$ system for comparison. For Pd-Ni system the calculated (solid line) and experimental values [41] (open circles) of the deviations of the lattice parameter from Vegard's law are in good agreement (Fig. 2(b)). It is seen from Fig. 2(b) that the lattice parameters are correctly predicted by EAM-WB potentials to within $<0.015 \AA$ and $<0.025 \AA$ for Pd-rich and Ni-rich alloys, respectively. Thus an application of the EAM-FBD and EAM-WB to the simulation of both the Ag$\mathrm{Ni}$ and Pd-Ni systems, respectively appears to be reasonable.

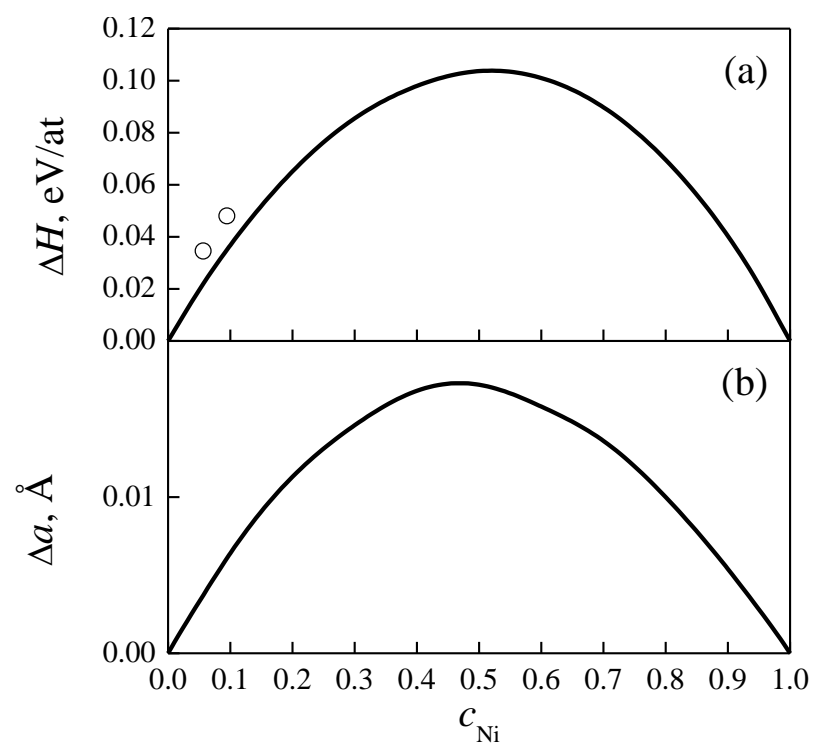

Fig. 1. Heat of solution $\Delta H$ (a) and deviation of the lattice parameter from Vegard's law $\Delta a$ (b) for bulk Ag-Ni system as a function of composition $c_{\mathrm{Ni}}$. Solid line: results using the EAM-FBD; open circles: experimental data [39] (a).

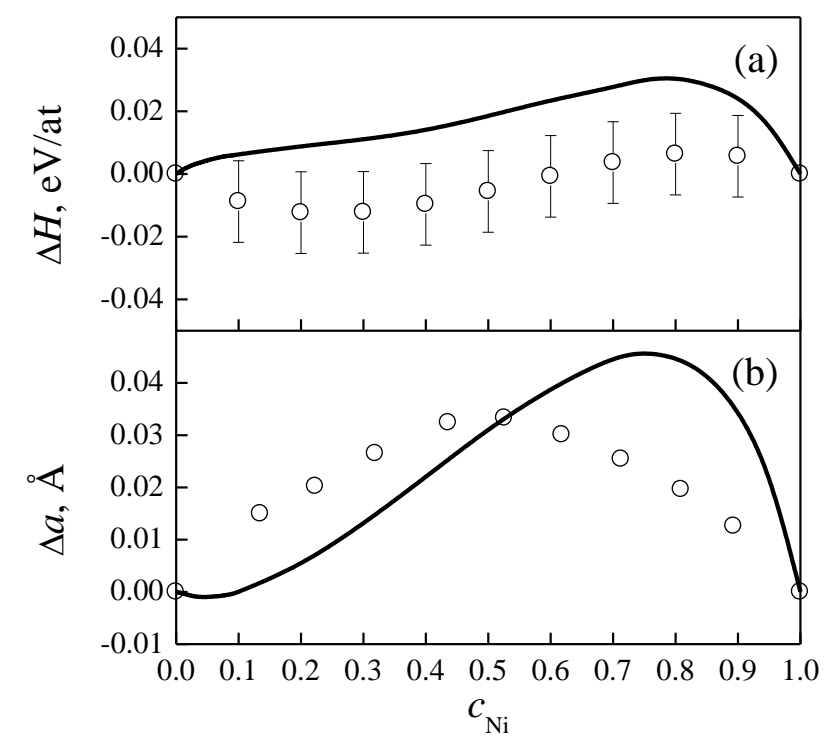

Fig. 2. Heat of solution $\Delta H$ (a) and deviation of the lattice parameter from Vegard's law $\Delta a$ (b) for bulk Pd-Ni system as a function of composition $c_{\mathrm{Ni}}$. Solid line: results using the EAM-WB; open circles: experimental data [40] (a) and [41] (b).

A spherical core-shell f.c.c. structure was chosen as the initial state for bimetallic $\mathrm{Ag}(\approx 28$ at. $\%)$ $-\mathrm{Ni}(\approx 72$ at. $\%)$ and $\mathrm{Pd}(\approx 66$ at. $\%)-\mathrm{Ni}(\approx 34$ at. $\%)$ nanoparticles. In the first case, a core with radius of $\sim 1.5 \mathrm{~nm}$ of $959 \mathrm{Ag}$ atoms was surrounded by a shell with external radius of $\sim 2.2 \mathrm{~nm}$ of $2504 \mathrm{Ni}$ atoms, while in the second case a core with radius of $\sim 1.4 \mathrm{~nm}$ of $1088 \mathrm{Ni}$ atoms was 
surrounded by a shell with external radius of $\sim 2.2 \mathrm{~nm}$ of $2132 \mathrm{Pd}$ atoms. Then the static relaxation procedure was applied to accommodate the core and shell atoms especially at the interface, since $\mathrm{Ag}$ atoms (the equilibrium bulk lattice parameter is $4.09 \AA$ ) and Pd atoms (the equilibrium bulk lattice parameter is $3.89 \AA$ ) are noticeably larger than $\mathrm{Ni}$ atoms (the equilibrium bulk lattice constant is $3.52 \AA$ ) with differences in atomic sizes of $\sim 15 \%$ and $\sim 10 \%$, respectively. Following this, atoms were given initial velocities according to the Maxwell distribution at the temperatures of $950 \mathrm{~K}$ for the Ag-Ni nanoparticle and $1000 \mathrm{~K}$ for the Pd-Ni nanoparticle and the MD procedure of isothermal annealing was performed. The MD procedure consisted of a numerical integration of the equations of atomic motion according to the Verlet algorithm [44] with a time step $\Delta t=1.5$ fs. During the simulation the momentum and a moment of momentum of the systems were conserved at zero values. Periodically, without an effect to the continuity of the annealing procedure, the systems were transferred to a state at $T=0 \mathrm{~K}$ where atoms occupied equilibrium positions in local potential minima by making use of the static relaxation method. After this, the atomic movements and structure transformations occurring in the models were analyzed.

\section{Results and discussion}

Let us first consider the results of the interdiffusion simulation in the Ag-Ni nanoparticle. As can be seen in Fig. 3(a), just after the static relaxation of the initial Ag-Ni nanoparticle model, the interface between the Ag-core and Ni-shell can be approximated by a spherical layer having a radius $15.5 \AA$. Actually, because of large lattice parameter mismatches, the interface is strongly distorted with quite a wide range of distances with the middle at a radius $\sim 15.5 \AA$. Representative snapshots from the MD simulation of interdiffusion in the Ag-core - Ni-shell nanoparticle during isothermal annealing at $T=950 \mathrm{~K}$ are given in Fig. 4 . It can be seen in Fig. 4 that $\mathrm{Ag}$ atoms diffuse quite rapidly through the $\mathrm{Ni}$-shell and begin to accumulate on the surface of the nanoparticle. The first $\mathrm{Ag}$ atoms coming to the surface of the nanoparticle reside preferentially at edges, vertices and high energies faces sites. Then the next Ag atoms coming to the surface of the nanoparticle meet the previous Ag atoms on the surface and form small clusters on the surface. However, this interdiffusion process becomes very slow and almost finishes when the average number of nearest silver neighbours of the Ag atoms on the surface reaches 2 . Thus, the Ag atoms coming from the core to the surface by diffusion through the Ni-shell form quite a dispersed single surface layer. On the other hand, a welldefined Ag core remains to occupy the centre of the nanoparticle. Shown in Fig. 3(b) the atomic radial distribution of the $\mathrm{Ag}$ and $\mathrm{Ni}$ atoms illustrates the separation of the structure into Ag-core - Ni-intermediate shell - Ag-disperse surface monolayer. It can be seen in Fig. 3(b) that only a few $\mathrm{Ag}$ atoms are in the intermediate shell of the nanoparticle.

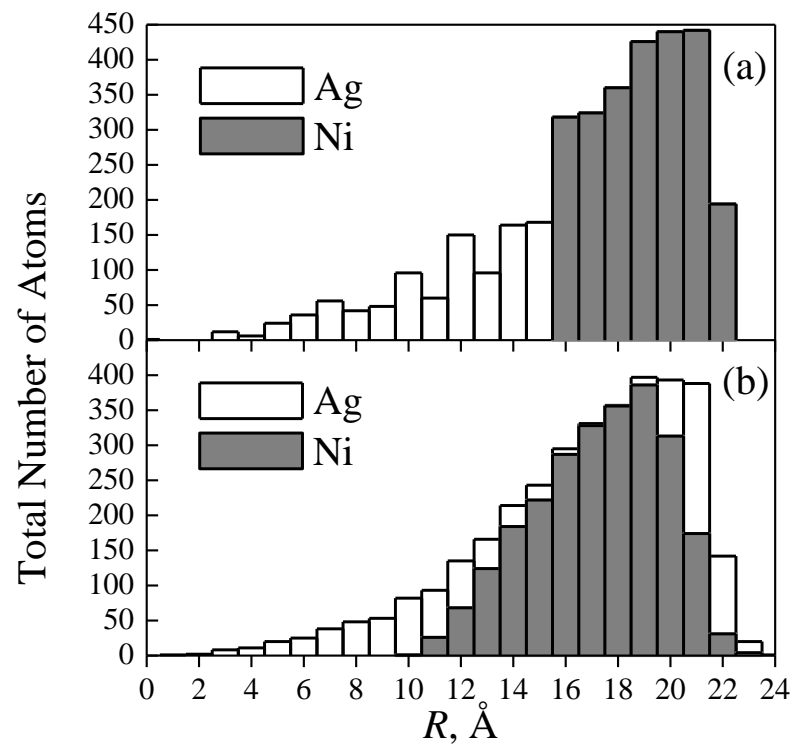

Fig. 3. Atomic radial distribution in the $\mathrm{Ag}-\mathrm{Ni}$ nanoparticle model at the initial time (a) and after the annealing at $T=950 \mathrm{~K}$ during $\sim 0.3 \mu \mathrm{s}(\mathrm{b})$.

These results are in good agreement with MD simulations of the growth of $\mathrm{Ag}-\mathrm{Ni}$ nanoparticles by deposition of $\mathrm{Ni}$ atoms onto a $\mathrm{Ag}$ core [18], where the final number of atoms in the system was $\sim 400$, which is one order of magnitude less than in our simulation. Thus, it is evident that the phenomenon of formation of a Ag-Ni-Ag surface-sandwich structure in Ag-Ni nanoparticles is quite general and apparently does not have a strong dependence on size. 

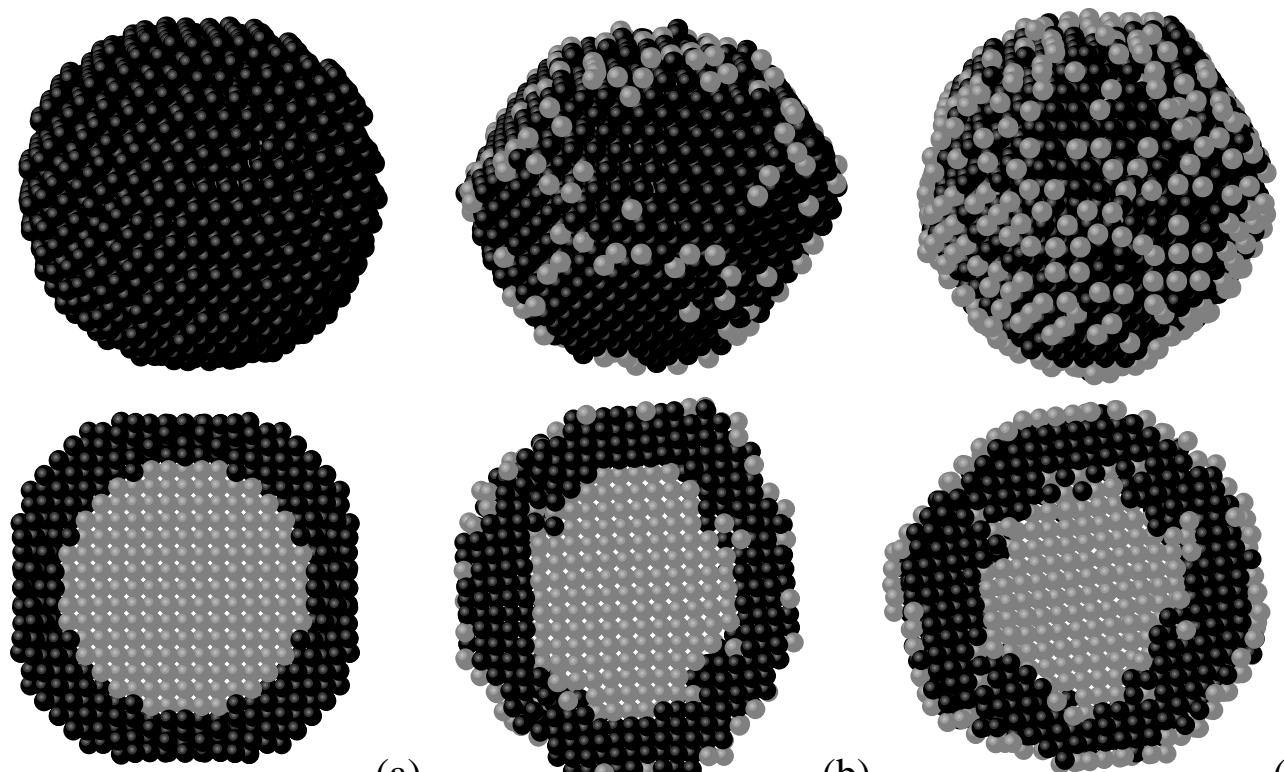

(a)
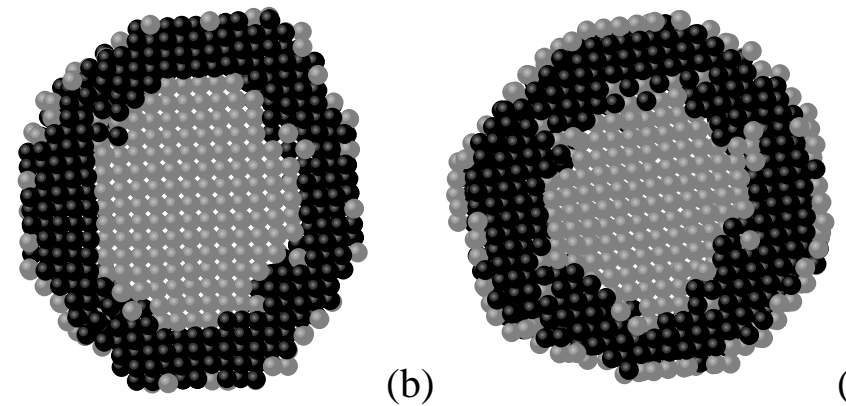

(c)

Fig. 4. Selected snapshots (after static relaxation) of the interdiffusion in the Ag-core - Ni-shell nanoparticle during annealing at $T=950 \mathrm{~K}$. Snapshots are taken at the beginning of the simulation (a) and after $\sim 0.03 \mu \mathrm{s} \mathrm{(b)} \mathrm{and} \sim 0.3 \mu \mathrm{s}$ (c). The surface and a central cross-section of the nanoparticle are shown in the top and bottom rows, respectively. $\mathrm{Ag}$ and $\mathrm{Ni}$ atoms are represented in gray and black, respectively.

In the case of the Pd-Ni nanoparticle, the initial interface between the $\mathrm{Ni}$-core and $\mathrm{Pd}$-shell can be approximated by a spherical layer with a radius $14.5 \AA$ (Fig. 5(a)). During the first $\sim 0.03 \mu$ s of MD annealing at $1000 \mathrm{~K}$, the distorted crystal order of the nanoparticle starts to be broken at the interface rather quickly, and then the structure of the whole nanoparticle evolves by interdiffusion to a disordered Pd-rich solid solution. Monitoring of the structure of the nanoparticle by the Voronoi polyhedron analysis shows a significant increase of the icosahedral short-range order during next annealing up to $\sim 0.5 \mu \mathrm{s}$. The centres of the icosahedral cages are almost always the smaller $\mathrm{Ni}$ atoms, surrounded by $4 \mathrm{Ni}$ and $8 \mathrm{Pd}$ atoms (see Fig. 6). The most favourable sites for $\mathrm{Ni}$ icosahedra are in the subsurface shell. Indeed, after $\sim 0.5 \mu$ s of the annealing, among the $790 \mathrm{Ni}$ atoms gone from the initial core to the shell, the icosahedra fraction is $\sim 78 \%$ whilst $\sim 52 \%$ of the $298 \mathrm{Ni}$ atoms remain in the core (see Figs.5(b) and 7).

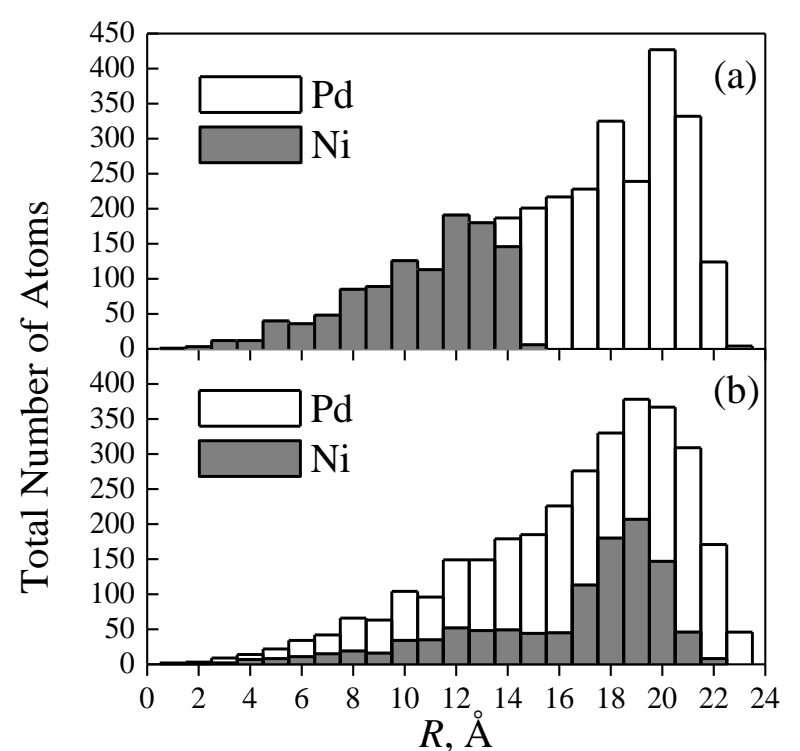

Fig. 5. Atomic radial distribution in the Pd-Ni nanoparticle model at the initial time (a) and after the annealing at $T=1000 \mathrm{~K}$ during $\sim 0.5 \mu \mathrm{s}(\mathrm{b})$.

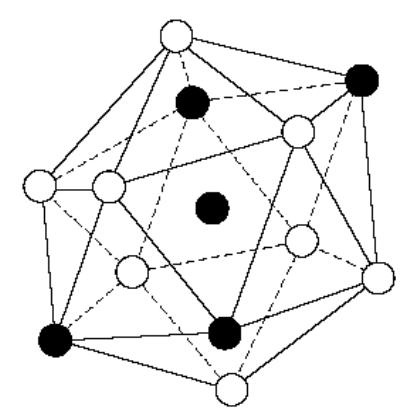

Fig. 6. The most preferable icosahedral surrounding of $\mathrm{Ni}$ atoms in the $\mathrm{Pd}-\mathrm{Ni}$ nanoparticle model: $4 \mathrm{Ni}$ and $8 \mathrm{Pd}$ nearest neighbours ( $\mathrm{Ni}$ is black, $\mathrm{Pd}$ is white).

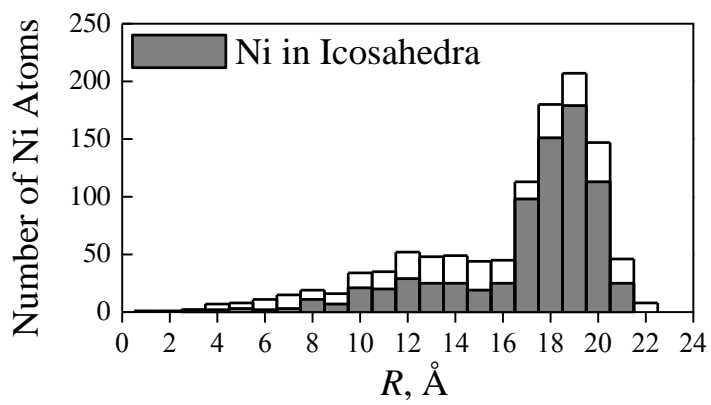

Fig. 7. Radial distribution of $\mathrm{Ni}$ atoms having an icosahedron as a coordination polyhedron in the Pd-Ni nanoparticle model after the annealing at $T=1000 \mathrm{~K}$ during $\sim 0.5 \mu$ s. 
It was established that $\mathrm{Ni}$ atoms coming by interdiffusion to the shell begin to accumulate in a layer just below the surface, and then locate in the centres of interpenetrating icosahedra in order to generate a subsurface layer (shell) as a Kagomé net (Fig. 8 (a)), which is almost completely covered by Pd atoms on both sides. We recall that planar layers of Kagomé nets are the basic structure of the well-known bulk Frank-Kasper phases of certain intermetallic alloys [45]. This subsurface Kagomé net shell of Ni atoms efficiently allows the minimization of the nanoparticle surface energy by increase of the average number of nearest neighbours of Pd surface atoms in comparison with the close-packed f.c.c. (111) surface. Indeed, the Ni Kagomé net layer $(K)$ is covered from each side by two Pd layers $\left(A^{\prime}\right.$ and $\left.A^{\prime \prime}\right)$, which can be obtained by splitting of a layer $A$ - close packed f.c.c. (111) layer (see Fig. 8 (b)). The forming of such a five-layer $A^{\prime} A^{\prime \prime} K A^{\prime \prime} A^{\prime}$ surface structure results in a third of the surface Pd atoms having increased their number of nearest neighbours from 9 (the close packed f.c.c. (111) surface) to 13 (see also [31]). The number of $\mathrm{Pd}$ atoms in each couple layers $A^{\prime}$ and $A^{\prime \prime}$ coincides with the number of $\mathrm{Ni}$ atoms in the Kagomé net layer, therefore, the total atomic fraction involved in the build up of the five-layer $A^{\prime} A^{\prime \prime} K A^{\prime \prime} A^{\prime}$ $\mathrm{Pd}_{2} \mathrm{Ni}$ ordering surface-sandwich structure in the nanoparticle with consideration of some imperfection of icosahedral order in Kagomé net layer can be estimated at $\sim 70 \%$. Thus, only 30\% atoms are located in the core of nanoparticle and form there a non-crystalline Pd-rich solid solution with quite strongly developed icosahedral short-range order. This final arrangement of the nanoparticle is the result of competition between the shell ( $70 \%$ atoms) and the core ( $\sim 30 \%$ atoms $)$ contributions to the excess energy.

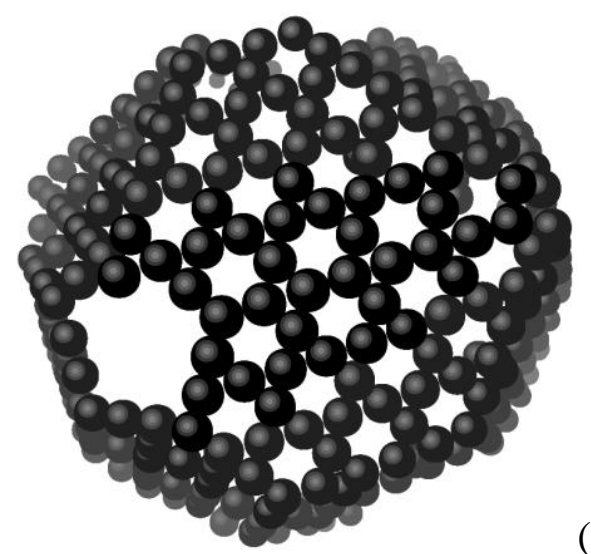

(a)

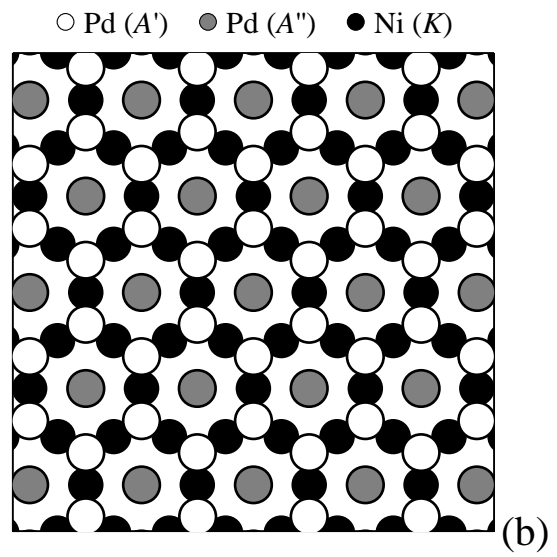

Fig. 8. (a) - Snapshot of the Ni subsurface shell having a structure of the Kagomé net with 'sequence faults' after $\sim 0.3 \mu \mathrm{s}$ of annealing at $T=1000 \mathrm{~K}$. The great majority of $\mathrm{Ni}$ atoms of the subsurface shell are located in the centres of interpenetrating icosahedra and have $4 \mathrm{Ni}$ and $8 \mathrm{Pd}$ nearest neighbours. For better clarity, only a half of the shell and only $\mathrm{Ni}$ atoms at centres of icosahedra are shown on the perspective projection with size and grey-scale graduation. (b) - Illustration of atomic positions of Pd and $\mathrm{Ni}$ atoms on a perfect fragment of surface structure $A^{\prime} A^{\prime \prime} K A^{\prime \prime} A^{\prime}$. Layers $A^{\prime}$ and $A^{\prime \prime}$ of $\mathrm{Pd}$ can be obtained by splitting of a layer $A$ - close packed f.c.c. (111) layer; layer $K$ of $\mathrm{Ni}$ is a Kagomé net layer.

Thus, our MD experiments demonstrate that under certain conditions a surface-sandwich segregation phenomenon at the nanoscale can be observed in systems with completely different phase diagrams in the bulk states: for example in systems displaying the extremely low mutual solubility as with the Ag-Ni system, or in systems exhibiting a continuous mutual solid solubility as with the Pd-Ni system.

\section{Summary}

On the basis of long-time scale molecular dynamics simulation in combination with the embedded atom method it has been demonstrated that interdiffusion in the initial f.c.c. Ag-core $(\approx 28$ at. \%) - Nishell $(\approx 72$ at. $\%)$ and $\mathrm{Ni}$-core $(\approx 34$ at. $\%)-\mathrm{Pd}$-shell $(\approx 66$ at. $\%)$ nanoparticles can result in surfacesandwich segregation. In the first case, we have observed the separation of the initial Ag-Ni core-shell structure in $\mathrm{Ag}$-core - Ni-intermediate shell - Ag-disperse surface monolayer. In the second case, the five-layer $A^{\prime} A^{\prime \prime} K A^{\prime \prime} A^{\prime} \mathrm{Pd}_{2} \mathrm{Ni}$ ordering surface-sandwich shell covers the core of a non-crystalline Pdrich solid solution with quite strongly developed icosahedral short-range order. In the shell $\mathrm{Ni}$ atoms are located in the centres of interpenetrating icosahedra to generate a subsurface layer, $K$, as a Kagomé net while the Pd atoms occupy the vertices of the icosahedra and coat this Ni layer from inside and outside by two Pd layers $A^{\prime}$ and $A^{\prime \prime}$, which can be obtained by splitting of a layer $A$ - close packed f.c.c. (111) layer. 
The results obtained allows us to conclude that under certain conditions a surface-sandwich segregation at the nanoscale can be observed in systems with completely different phase diagrams in the bulk states.

In addition, we can suggest experiment that would be interesting: a study of the formation of the fivelayer $A^{\prime} A^{\prime \prime} K A^{\prime \prime} A \mathrm{Pd}_{2} \mathrm{Ni}$ ordering surface-sandwich structure in well-annealed (111) oriented ( $\left.A B C A B C \ldots\right)$

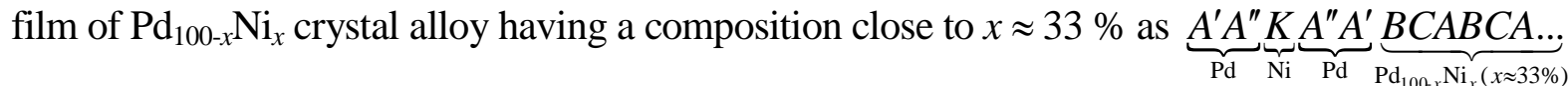

\section{Acknowledgements}

This research was supported by the Australian Research Council. One of us (E.V.L.) wishes to thank the University of Newcastle for the award of a Research Fellowship.

\section{References}

[1] N. Toshima, M. Kanemaru, Y. Shiraishi, Y. Koga: J. Phys. Chem B Vol. 109 (2005), p. 16326.

[2] D. Chen, W. Wang, S. Huang: J.Phys.: Conmdens Matter Vol. 19 (2007), p. 356217.

[3] M. Gaudry, E. Cottancin, M. Pellarin, J.Lerme et.al: Phys. Rev. B Vol 67 (2003), p. 155409.

[4] J. Park, J. Joo, S.G. Kwon, Y. Jang et.al: Angew. Chem. Int. Ed. Vol. 46 (2007), p. 4630.

[5] T. Bala, S.D. Bhame, P.A. Joy, B.L.V. Prasad et.al: J. Mater. Chem. Vol. 14 (2004), p.2941.

[6] C.C. Lee, D.H. Chen: Nanotechnology Vol. 17 (2006), p.3094.

[7] D.H. Chen, S-R. Wang: Mater Chem. And Phys. Vol. 100 (2006), p.468.

[8] S. Takenaka, Y. Shigeta, E. Tanabe, K. Otsuka: J. Phys. Chem. Vol. 108 (2004), p.7656.

[9] S. Sao-Joao, S. Giorgio, J.M. Penisson, C. Chapon et.al: J. Phys. Chem. Vol. 109 (2005), p.342.

[10] A.B. Hungría, J.J. Calvino, J.A. Anderson, A. Martínez-Arias: Appl. Catal. Vol. 62 (2006), p.359.

[11] P. Miegge, J.L. Rousset, B. Tardy, J. Massardier et.al: J. Catal. Vol. 149 (1994), p.404.

[12] P. Herman, J.M. Guigner, B. Tardy, Y.Jugnet et.al: J. Catal. Vol. 163 (1996), p. 169.

[13] A.C. Michel, L. Lianos, J.L. Rousset, P. Delichère et.al: Surf. Sci. Vol. 416 (1998), p.288.

[14] L. Porte, M. Phaner-Goutorbe, J.M. Guigner, J.C. Bertolini: Surf. Sci. Vol. 424 (1999), p.262.

[15] O.M. Løvvik: Surf. Sci. Vol. 583 (2005), p. 100.

[16] G. Rossi, A. Rappalo, C. Mottet. A. Fortunelli et.al: Phys. Rev. Lett Vol. 93 (2004), p. 105503.

[17] A. Rapallo, G. Rossi, R. Ferrando, A. Fortunelli et. al: J. Chem. Phys. Vol. 122 (2005), p. 194308.

[18] F. Baletto, C. Mottet, R. Ferrando: Phys. Rev. Lett. Vol. 90 (2003), p. 135504.

[19] F. Baletto, C. Mottet, A. Rappalo, G. Rossi et. al: Surf. Sci. Vol.566-568 (2004), p. 192.

[20] G.N. Derry, C.B. McVey, P.J. Rous: Surf. Sci. Vol. 326 (1995), p.59.

[21] M. Abel, Y. Robach, J.C. Bertolini, L. Porte: Surf. Sci. Vol.454-456 (2000), p 1.

[22] G.N. Derry, R. Wa: Surf. Sci. Vol.566 (2004), p. 862.

[23] J.L. Rousset, J.C. Bertolini, P. Miegge: Phys. Rev. B Vol. 53 (1996), p.4947.

[24] B.C. Khanra, J.C. Bertolini, J.L. Rousset: J. Mol. Catal. A Vol. 129 (1998), p.233.

[25] A. Christensen, A.V. Ruban, H.L. Skriver: Surf. Sci. Vol. 383 (1997), p.235.

[26] L.V. Poyurovskii, A.V. Ruban, I.A. Abrikosov, Y.K. Vekilov et.al: JEPT Lett. Vol. 73 (2001), p.415.

[27] J.-S. Filhol, D. Simon, P.Sautet: Phys. Rev. B Vol. 64 (2001), p. 85412.

[28] S. Helfensteyn, J. Luyten, L. Feyaerts, C. Creemers: Appl. Surf. Sci. Vol 212 (2003), p. 844.

[29] A.A. Dmitriev, A.V. Evteev, V.M. Ievlev, A.T. Kosilov: Doklady Physics Vol.49 (2004), p. 292.

[30] M. Mavrikakis: Nature Mater.Vol 5 (2006), p. 847.

[31] E.V. Evteev, E.V. Levchenko, I.V. Belova, G.E. Murch: Def. Diff. Forum Vol. 277 (2008), p. 207.

[32] M.S. Daw and M.I. Baskes: Phys. Rev. B Vol. 29 (1984), p. 6443.

[33] M.S. Daw: Phys. Rev. B Vol. 39 (1989), p. 7441.

[34] S.M. Foiles, M.I. Baskes, M.S. Daw: Phys. Rev. B Vol. 33 (1986), p. 7983.

[35] S.M. Foiles: Phys. Rev. B Vol. 32 (1985), p. 3409.

[36] S.M. Foiles, J.B. Adams: Phys. Rev. B Vol. 40 (1989), p. 590909.

[37] W.R. Tyson, W.A. Miller: Surf. Sci. Vol. 62 (1977), p. 267.

[38] S.M. Foiles: Phys. Rev. B Vol. 32 (1985), p. 7685.

[39] H.G. Zolla, F. Spaepen: Acta Mater. Vol. 47 (1999), p. 2391.

[40] R. Hultgren, P.D. Desai, D.T. Hawkins, M. Gleiser, K.K. Kelly: Selected Values of the Thermodynamic Properties of Binary Alloys (American Society for Metals, Metals Park, Ohio 1973).

[41] L.R. Bidwell, R. Speiser: Acta Cryst. Vol. 17 (1964), p. 1473.

[42] G. Tammann, W. Oelson: Z. Anorg. Chem. Vol. 186 (1930), p. 264.

[43] J. Ladet, J. Berradini, F. Cabane-Brouty: Scr. Metall. Vol. 10 (1976), p. 195.

[44] L. Verlet: Phys. Rev. Vol. 159 (1967), p. 98.

[45] F.C. Frank and J.S. Kasper: Acta Cryst. Vol. 11 (1958), p. 184; Vol. 12 (1959), p. 483. 
Diffusion in Materials - DIMAT2008

doi:10.4028/www.scientific.net/DDF.289-292

Surface-Sandwich Segregation Phenomena in Bimetallic Ag-Ni and Pd-Ni

Nanoparticles: A Molecular Dynamics Study

doi:10.4028/www.scientific.net/DDF.289-292.657

\section{References}

[1] N. Toshima, M. Kanemaru, Y. Shiraishi, Y. Koga: J. Phys. Chem B Vol. 109 (2005), p. 16326.

doi:10.1021/jp051400h

PMid: 16853075

[2] D. Chen, W. Wang, S. Huang: J.Phys.: Conmdens Matter Vol. 19 (2007), p. 356217. doi:10.1088/0953-8984/19/35/356217

[3] M. Gaudry, E. Cottancin, M. Pellarin, J.Lerme et al: Phys. Rev. B Vol 67 (2003), p. 155409.

doi:10.1103/PhysRevB.67.155409

[4] J. Park, J. Joo, S.G. Kwon, Y. Jang et al: Angew. Chem. Int. Ed. Vol. 46 (2007), p.4630. doi:10.1002/anie.200603148

[5] T. Bala, S.D. Bhame, P.A. Joy, B.L.V. Prasad et al: J. Mater. Chem. Vol. 14 (2004), p.2941.

doi:10.1039/b405335b

[6] C.C. Lee, D.H. Chen: Nanotechnology Vol. 17 (2006), p.3094.

doi:10.1088/0957-4484/17/13/002

[7] D.H. Chen, S-R. Wang: Mater Chem. And Phys. Vol. 100 (2006), p.468.

doi:10.1016/j.matchemphys.2006.01.027

[8] S. Takenaka, Y. Shigeta, E. Tanabe, K. Otsuka: J. Phys. Chem. Vol. 108 (2004), p.7656.

doi:10.1021/jp0377331

[9] S. Sao-Joao, S. Giorgio, J.M. Penisson, C. Chapon et al: J. Phys. Chem. Vol. 109 (2005), p.342.

doi:10.1021/jp040473i

PMid: 16851020

[10] A.B. Hungría, J.J. Calvino, J.A. Anderson, A. Martínez-Arias: Appl. Catal. Vol. 62 (2006), p.359.

doi:10.1016/j.apcatb.2005.09.003 
[11] P. Miegge, J.L. Rousset, B. Tardy, J. Massardier et al: J. Catal. Vol. 149 (1994), p.404. doi:10.1006/jcat.1994.1307

[12] P. Herman, J.M. Guigner, B. Tardy, Y.Jugnet et al: J. Catal. Vol. 163 (1996), p. 169. doi:10.1006/jcat.1996.0316

[13] A.C. Michel, L. Lianos, J.L. Rousset, P. Delichère et al: Surf. Sci. Vol. 416 (1998), p.288.

doi:10.1016/S0039-6028(98)00614-1

[14] L. Porte, M. Phaner-Goutorbe, J.M. Guigner, J.C. Bertolini: Surf. Sci. Vol. 424 (1999), p.262.

doi:10.1016/S0039-6028(99)00020-5

[15] O.M. Løvvik: Surf. Sci. Vol. 583 (2005), p. 100.

doi:10.1016/j.susc.2005.03.028

[16] G. Rossi, A. Rappalo, C. Mottet. A. Fortunelli et al: Phys. Rev. Lett Vol. 93 (2004), p. 105503.

doi:10.1103/PhysRevLett.93.105503

PMid:15447416

[17] A. Rapallo, G. Rossi, R. Ferrando, A. Fortunelli et al: J. Chem. Phys. Vol. 122 (2005), p. 194308.

doi:10.1063/1.1898223

PMid:16161574

[18] F. Baletto, C. Mottet, R. Ferrando: Phys. Rev. Lett. Vol. 90 (2003), p. 135504. doi:10.1103/PhysRevLett.90.135504

PMid:12689304

[19] F. Baletto, C. Mottet, A. Rappalo, G. Rossi et al: Surf. Sci. Vol.566-568 (2004), p. 192. doi:10.1016/j.susc.2004.05.044

[20] G.N. Derry, C.B. McVey, P.J. Rous: Surf. Sci. Vol. 326 (1995), p.59. doi:10.1016/0039-6028(94)00724-1

[21] M. Abel, Y. Robach, J.C. Bertolini, L. Porte: Surf. Sci. Vol.454-456 (2000), p 1. doi:10.1016/S0039-6028(00)00269-7

[22] G.N. Derry, R. Wa: Surf. Sci. Vol.566 (2004), p. 862.

doi:10.1016/j.susc.2004.06.022

[23] J.L. Rousset, J.C. Bertolini, P. Miegge: Phys. Rev. B Vol. 53 (1996), p.4947. doi:10.1103/PhysRevB.53.4947 
[24] B.C. Khanra, J.C. Bertolini, J.L. Rousset: J. Mol. Catal. A Vol. 129 (1998), p.233. doi:10.1016/S1381-1169(97)00150-7

[25] A. Christensen, A.V. Ruban, H.L. Skriver: Surf. Sci. Vol. 383 (1997), p.235.

doi:10.1016/S0039-6028(97)00177-5

[26] L.V. Poyurovskii, A.V. Ruban, I.A. Abrikosov, Y.K. Vekilov et al: JEPT Lett. Vol. 73 (2001), p.415.

[27] J.-S. Filhol, D. Simon, P.Sautet: Phys. Rev. B Vol. 64 (2001), p. 85412.

doi:10.1103/PhysRevB.64.085412

[28] S. Helfensteyn, J. Luyten, L. Feyaerts, C. Creemers: Appl. Surf. Sci. Vol 212 (2003), p. 844.

doi:10.1016/S0169-4332(03)00088-6

[29] A.A. Dmitriev, A.V. Evteev, V.M. levlev, A.T. Kosilov: Doklady Physics Vol.49 (2004), p. 292.

doi:10.1134/1.1763619

[30] M. Mavrikakis: Nature Mater.Vol 5 (2006), p. 847.

doi:10.1038/nmat 1767

PMid:17077844

[31] E.V. Evteev, E.V. Levchenko, I.V. Belova, G.E. Murch: Def. Diff. Forum Vol. 277

(2008), p. 207.

doi:10.4028/www.scientific.net/DDF.277.207

[32] M.S. Daw and M.I. Baskes: Phys. Rev. B Vol. 29 (1984), p. 6443.

doi:10.1103/PhysRevB.29.6443

[33] M.S. Daw: Phys. Rev. B Vol. 39 (1989), p. 7441.

doi:10.1103/PhysRevB.39.7441

[34] S.M. Foiles, M.I. Baskes, M.S. Daw: Phys. Rev. B Vol. 33 (1986), p. 7983.

doi:10.1103/PhysRevB.33.7983

[35] S.M. Foiles: Phys. Rev. B Vol. 32 (1985), p. 3409.

doi:10.1103/PhysRevB.32.3409

[36] S.M. Foiles, J.B. Adams: Phys. Rev. B Vol. 40 (1989), p. 590909.

[37] W.R. Tyson, W.A. Miller: Surf. Sci. Vol. 62 (1977), p. 267.

doi:10.1016/0039-6028(77)90442-3

[38] S.M. Foiles: Phys. Rev. B Vol. 32 (1985), p. 7685. 
doi:10.1103/PhysRevB.32.7685

[39] H.G. Zolla, F. Spaepen: Acta Mater. Vol. 47 (1999), p. 2391.

doi:10.1016/S1359-6454(99)00131-7

[40] R. Hultgren, P.D. Desai, D.T. Hawkins, M. Gleiser, K.K. Kelly: Selected Values of the Thermodynamic Properties of Binary Alloys (American Society for Metals, Metals Park, Ohio 1973).

[41] L.R. Bidwell, R. Speiser: Acta Cryst. Vol. 17 (1964), p. 1473.

doi:10.1107/S0365110X64003632

[42] G. Tammann, W. Oelson: Z. Anorg. Chem. Vol. 186 (1930), p. 264.

[43] J. Ladet, J. Berradini, F. Cabane-Brouty: Scr. Metall. Vol. 10 (1976), p. 195.

doi:10.1016/0036-9748(76)90148-4

[44] L. Verlet: Phys. Rev. Vol. 159 (1967), p. 98.

doi:10.1103/PhysRev.159.98

[45] F.C. Frank and J.S. Kasper: Acta Cryst. Vol. 11 (1958), p. 184; Vol. 12 (1959), p. 483. 